\title{
N-back based Working Memory Contents to Improve Cognitive Function
}

\author{
Dong-Yeon Kim ${ }^{1}$, Sung-Wook Shin ${ }^{2}$ and Sung-Taek Chung ${ }^{3}$ * \\ ${ }^{1,2,3}$ Dept. of Computer Engineering Korea Polytechnic University \\ ${ }^{1}$ kdy2917@naver.com, ${ }^{2}$ napalza@kpu.ac.kr, ${ }^{3}$ unitaek@kpu.ac.kr
}

\begin{abstract}
Cognitive function damage can cause various problems such as work performance requiring concentration and independent daily life as well as social life which requires interpersonal relationship. In the cognitive function area, the working memory is a function to recreate only the information necessary for the external information and the memory of the past, and utilize it appropriately for the daily life behavior, thus working memory training helps improve the cognitive function of the elderly or children with weakened cognitive function. This paper employed an n-back method, one of the frequently mentioned methods in working memory training studies recently, as the basic program of research and established working memory training contents. This content is designed not only to select the difficulty level and four basic n-back training methods by visual (location, color) or auditory (alphabet, number) stimulation, but also to make training by creating $n$-back based functional game which can induce user's interest.
\end{abstract}

Keywords: Cognitive; Working Memory; N-back; Contents

\section{Introduction}

Cognitive function refers to a series of brain procedures to process diverse information. If the cognitive function is damaged by brain diseases, or others, the person experiences difficulties in his or her working function, daily activities and interpersonal relationships and can hardly perform ADL (Activities of Daily Living) independently [1]. Cognitive function damage is treated under rehabilitation therapy and the significance of cognitive function training has been continuously studied by many researchers. These studies on cognitive function training most frequently mention about working memory that temporarily maintains and manipulates necessary information [2]. In general, working memory is different from short-term memory which stores temporary information, it is different in not only storing temporary information but implementing information selection, use, and others [3]. In other words, it is closely related to the high level cognitive activities of linguistic understanding, writing, speaking, reasoning and hypothesis building as well as daily cognitive activities such as calculating small change [4-7]. Therefore, working memory deterioration means cognitive function weakening. It can be said that the stronger the working memory, the higher the person's cognitive function is. Thus, the degree of cognitive functional damage can be assessed by testing one's working memory. Also, if systematic working memory training is performed in daily lives continuously, it would be deeply useful in improving the cognitive function of patients with weakened cognitive function due to geriatric diseases including stroke and dementia and those with cognitive functional problems such as language disorder, learning difficulties, ADHD (Attention Deficit Hyperactivity Disorder), and mental retardation [8-10].

Received (May 23, 2017), Review Result (August 25, 2017), Accepted (September 20, 2017) 
OSPAN (Operation Span Task) and n-back are major indicators used in working memory training or measurement. OSPAN presents mathematical processes and words together for test takers to distinguish right answers from wrong answers then remember the presented words [11-12]. In general, since OSPAN requires mathematical processing, test takers with lower level educational background may have difficulty in solving them. Children may also lose their interest in the test as they have to solve math questions.

N-back is a working memory training method which sees if a currently presented stimulation matches with the stimulation appeared $n$ turns earlier when stimuli are presented consecutively. N-back has a simple structure easy for anyone to participate regardless of his or her academic background or age. The stimuli used in n-back test include visual stimuli such as location and forms along with auditory stimuli such as the voice reading of numbers, alphabets, etc. Training based on visual stimuli helps mainly activate selective attention while training based on auditory stimuli activates the central executive area that controls attention in working memory [13].

Most of the n-back related programs developed in preceding studies or available on the market are in the matrix of $3 \times 3$ blocs A random bloc, excluding the one in the center, changes to show a visual stimulus or numbers, alphabets, etc. are read aloud verbally or sounded through audio to give an auditory stimulus for test takers to assess if they match with each other [14]. Previously, n-back contents were presented regardless of participants' age, and degree of brain diseases and did not contain diversified training programs. A new method to improve cognitive function is necessary which can be customized for different users depending upon their ability levels by selecting n-back related auditory and visual stimuli in training. In other words, users' training achievement could be enhanced by integrating diverse kinds of n-back contents, customizing them according to user ability and providing feedback on their training results.

In this paper, basic n-back training programs and n-back game contents were developed, whose visual and auditory stimuli are adjustable by users. In addition, the proposed program was made to inform the trend of changes in users' cognitive function status in relation to their training results during training so that they could choose an appropriate content difficulty level for themselves.

\section{Methods}

\subsection{N-Back Training}

N-back is used as an experimental tool in studies on working memory training and measurement or cognitive load characteristics[15]. Recently, n-back receives increasing attention as a research method related to cognitive function based on the study finding that it has a strong correlation with the spatial reasoning task of RAPM(Raven's Advanced Progressive Matrices) as well[14]. Generally, n-back is implemented by presenting a random visual or auditory stimulus then letting participants see if the present stimulation is the same as the stimulation presented $\mathrm{n}$ turns back. Figure 1. shows 2-back, 3-back and 4-back implementation processes with spatial stimulation mainly used in previous studies. 2-back tests if the present stimulation corresponds with the stimulation presented 2 turns back. Therefore, in the 2-back shown in Figure 1. The initial judgment process is triggered upon the presentation of the 3rd stimulus and the training proceeds continuously until a judgment error takes place. In the 3-back training, judgment begins with the 4th stimulation presentation and, in the 4-back, it starts upon the 5th stimulation presentation. N-back training difficulty levels can be increased by adding heavier cognitive load to participants depending upon the number of visual and auditory stimulation types and size of $n$. 


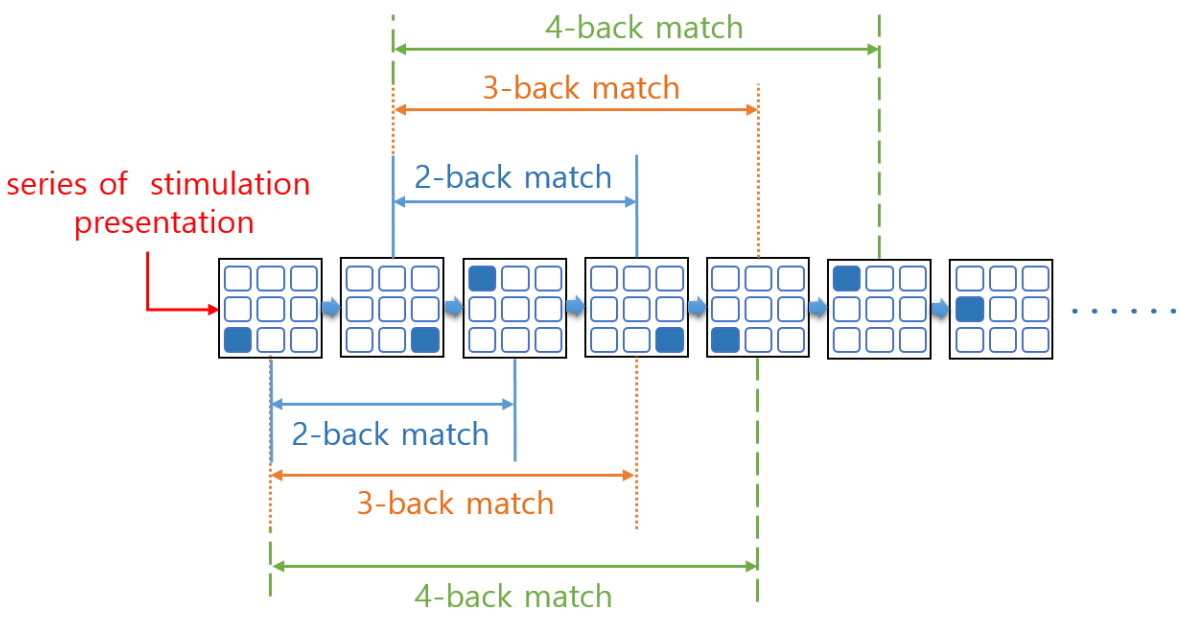

Figure 1. N-Back Task Process

\subsection{Working Memory Training Contents}

The working memory training contents in this study integrated the existing n-back training methods into one single content to be used in training according to users' choice. Feedback on their results is provided to help them feel the sense of achievement and motivation during training, encouraging users' voluntary training participation. Moreover, the n-back-based series game was designed in this study to promote proactive user participation while keeping the program fun and interesting for users so they do not become bored easily. The developed contents of this study follow the n-back stimulation method presented in the previous study. They give new stimuli at 2.5 second intervals by repeating the presentation of visual or auditory stimulus for 0.5 seconds[16]. Users can select stimulation types they desire among the visual stimuli of position or color and auditory stimuli of the sound of reading aloud numbers or alphabets. By doing so, they customize training contents for themselves. Task difficulties are structured in 3 levels and adjustable selectively depending upon individual user's training results.

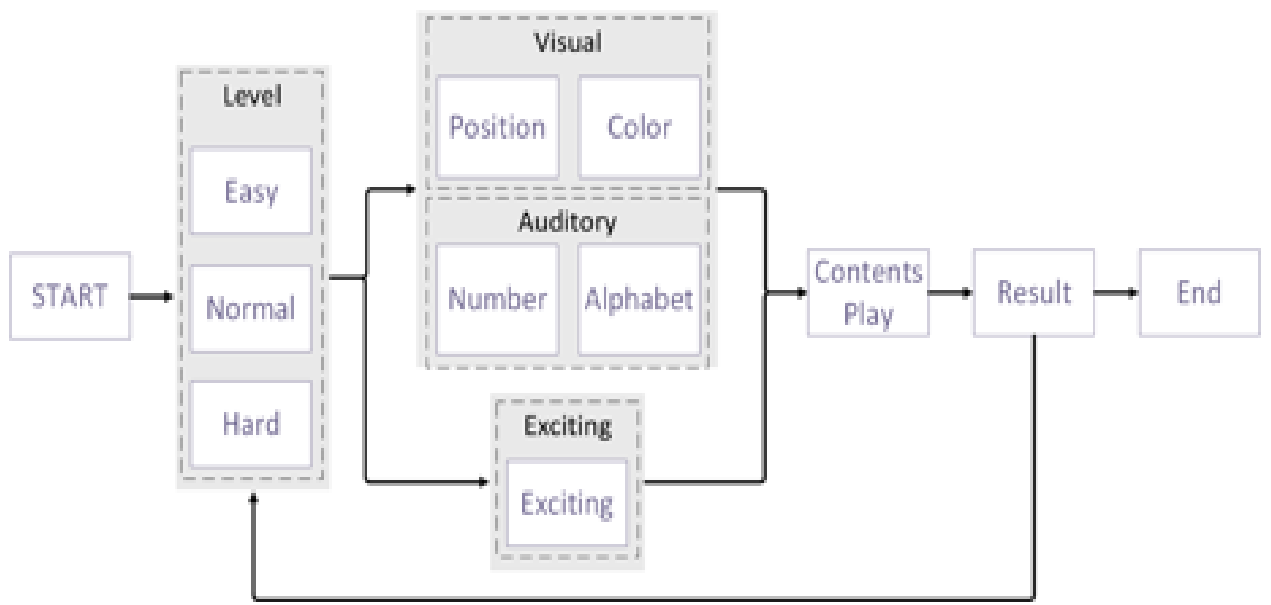

Figure 2. Flow of Working Memory Training Content

Figure 2 shows the flow of working memory training content. Upon the start of the content, users can choose the difficulty level they wish then select to proceed n-back training either based on Auditory/Visual or Exciting. If the auditory stimulation is chosen, sound stimuli of numbers and alphabets are given in the training. If the visual stimulation is chosen, position and color stimuli are shown in the training process. Exciting is to 
choose an n-back-based game content and the training is implemented without any further options to choose. All of the contents show the result page to users after training completion for them to check their results and choose either to receive training once again or finish the program.

The program was developed using the development instrument of Unity 5 in the Window 7 operating system environment. Display device was basically a PC monitor and Android-based smart device screen. To present voice stimuli in the training, the PC needed to have a separate device for voice output and the smartphone provided sound through own speaker.

\section{Contents Implementation}

\subsection{Contents Select Mode Pages}

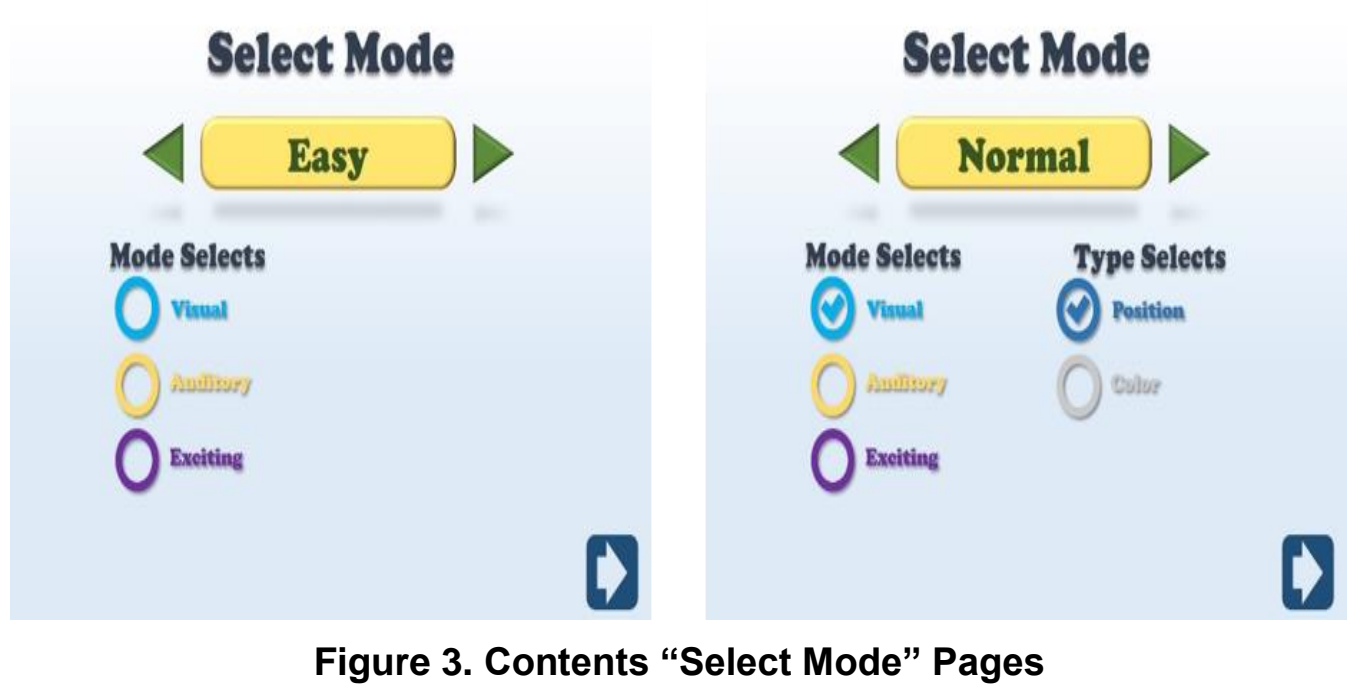

Figure 3 shows the program pages where users can select the training difficulty and type they want after program execution. Users can click the setting button in the middle of the screen to the left or right to adjust the difficulty. Difficulty levels are in three categories - Easy, Normal and Hard. Tables 1 and 2 show the n-back training type and a number of stimuli according to content difficulty. Table 1 shows n-back training types according to the difficulty levels of visual and auditory stimuli. The Easy mode provides 2-back training; Normal, 3-back; and Hard, 4-back. The maximum number of stimulation trials is 22 in Easy; 23, in Normal; and 24 in Hard. Stimuli are given at most 20 times in all of the cases to test at most 20 times of matching trials. The Exciting mode was designed using game elements to train users' working memory based on space stimuli while keeping them interested and immersed. Table 2 demonstrates that the more difficult the content level is, the more diversified the types of space stimuli and the more various the n-back types to implement become. 
Table 1. Difficulty Levels According to Visual and Auditory Stimulation

\begin{tabular}{|c|c|c|c|}
\hline Level & Max. No. of Trial & $\begin{array}{c}\text { Max. No. of } \\
\text { Judgments }\end{array}$ & n-back \\
\hline Easy & 22 & 20 & $2-$ \\
\hline Normal & 23 & 20 & $3-$ \\
\hline Hard & 24 & 20 & $4-$ \\
\hline
\end{tabular}

Table 2. Game Content Levels in "Exciting" Mode

\begin{tabular}{|c|c|c|}
\hline Level & Type of Position & n-back \\
\hline Easy & 4 & $1-/ 2-$ \\
\hline Normal & 5 & $1-/ 2-/ 3-$ \\
\hline Hard & 6 & $1-/ 2-/ 3-/ 4-$ \\
\hline
\end{tabular}

\subsection{N-Back Contents According to Visual/Auditory Stimulation}

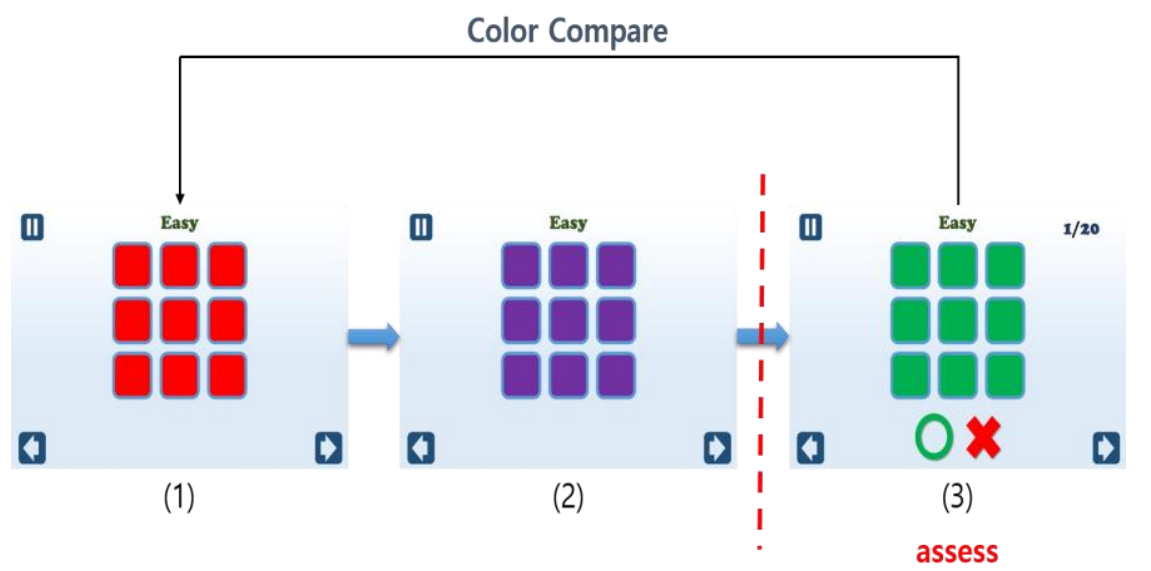

(a) Contents "Select Mode" Pages

Position Compare

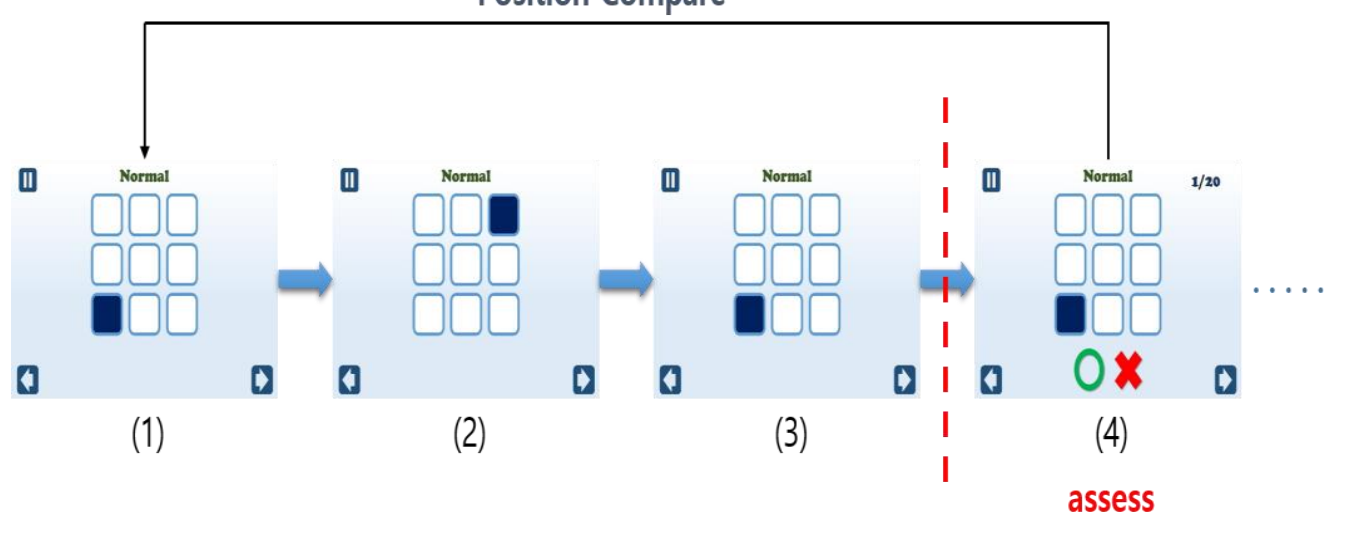

(b) Contents "Select Mode" Pages 


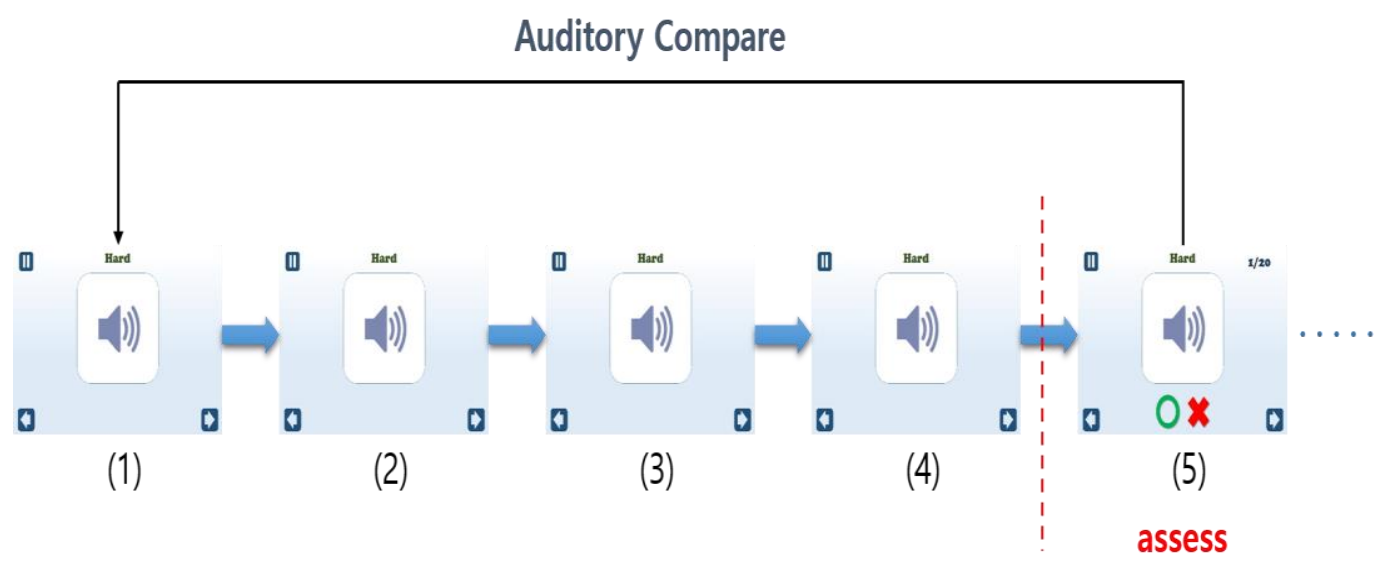

(c) Contents "Select Mode" Pages

Figure 4. Visual or Auditory Stimulation in Each Difficulty Level

Figure 4 shows n-back training contents supported by visual or auditory stimulation. Among the 8 different stimuli in each category of color, position and voice, stimuli are presented consecutively one by one according to difficult levels. If the present given stimulus matches with an earlier stimulus, users click the ' $O$ ' button appearing in the lower part of the screen. If not, they can click ' $X$ '. Content progress status is shown in the upper right part of the screen. To change the training content types or difficulty levels, users can click the arrow in the lower left part of the screen to move to the content selection page. Also, if a user wants to pause the training for a while, he or she can click the stop button in the upper left part.

Visual stimulation utilizes colors in training. Eight different colors (red, orange, yellow, green, blue, purple and black) are presented in the screen. Users are required to judge if the present color matches with the color shown $n$ turns back in each difficulty level they chose. Figure 4 (a) shows the color-based training in the Easy mode, which is executed in order of (1)-(2)-(3). In Table, 1, since 2-back training is implemented at the Easy level, the initial judgement begins at (3) screen. Once judgment begins, the screen shows training progress status and ' $\mathrm{O}$ ' and ' $\mathrm{X}$ ' buttons. By using these, users can judge about the matching and see where their training process stands. In Figure 4. (a), for instance, (1) and (3) have different colors. Thus, a user should click ' $X$ ' to get the right answer. If he or she clicks ' $\mathrm{O}$ ', it will be the wrong answer.

The working memory training on position stimulation was developed in reference to the existing studies on working memory and n-back [16-17]. The training method is to change the positions of blocs arranged in $3 \times 3$ matrix excluding the one in the center for users to compare the presently shown bloc position with that of the bloc shown $n$ turn earlier. Figure 4 (b) is the progress of training content on position stimulation in the Normal mode. Since 3-back training is implemented in this case, bloc positions are presented on order of (1)-(2)-(3)-(4) and users begin their judgment at (4). In Figure 4 (b), since (1) and (4) have the same positions, users should click ' $O$ ' to get the right answer. If they click ' $\mathrm{X}$ ', they get the wrong answer.

Figure 4 (c) is on a working memory training content based on auditory stimulation in the Hard mode. It shows the process of giving voice stimuli of alphabets and numbers. Eight auditory stimuli of alphabets are a, b, c, d, e, f, g, and h and auditory number stimuli are 1, 2, 3, 4, 5, 6, and 7. As indicated in Figure 4. (c), the content is implemented in order of (1)-(2)-(3)-(4)-(5). It is the 4-back training method and users compare the auditory stimulus (5) with (1) to assess if they match together. For instance, if (1) was the voice stimulation of alphabet ' $a$ ' and (5) was that of ' $d$ ', the two do not correspond with each other and users should click ' $X$ ' to get the correct answer. If they get a wrong answer, the 
training is terminated. If they click no wrong answer, the training continues up to until 20 trials of matching judgment until completion.

\subsection{N-back-based Exciting Game Contents}

The Exciting mode was designed as an n-back-based game content that can interest users with position stimuli during their gaming and training process. Figure 5. shows the content progress and the program is implemented in order of Trial 1, Trial 2, Trial 3 and so forth. Users can follow n-back training for one round in each Trial. In Figure 5., Trial 1 is completed after executing one round of 2-back training. The subsequent Trial provides 1-back or 2-back training. If a user gets a wrong answer, the corresponding Trial is terminated and the program shows the result page. Such a method is expected to help users see their ability to react corresponding tasks based on random n-back presentation.

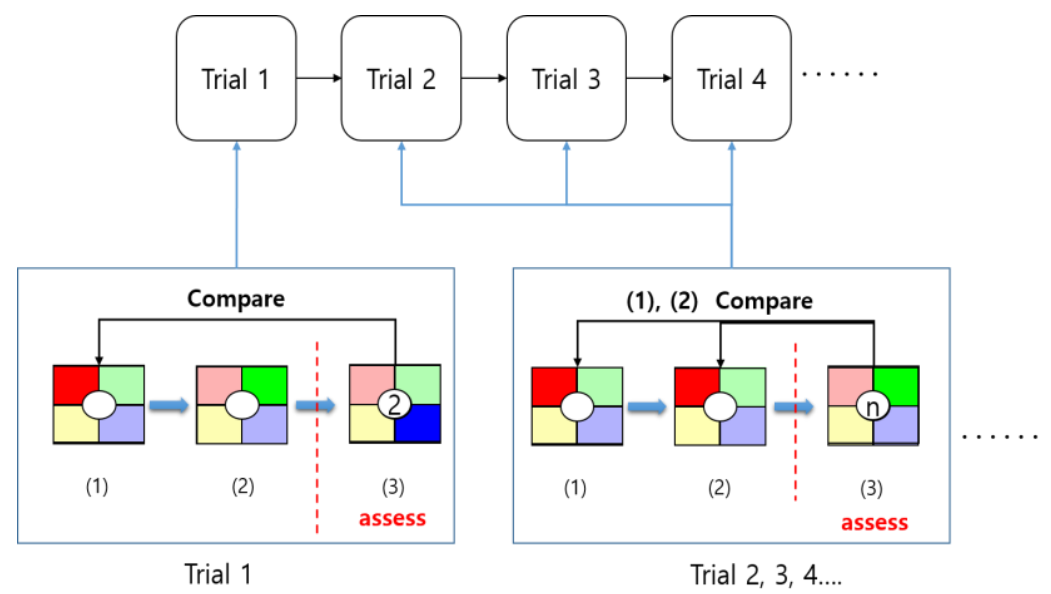

Figure 5. Content Progress in the "Easy" Mode

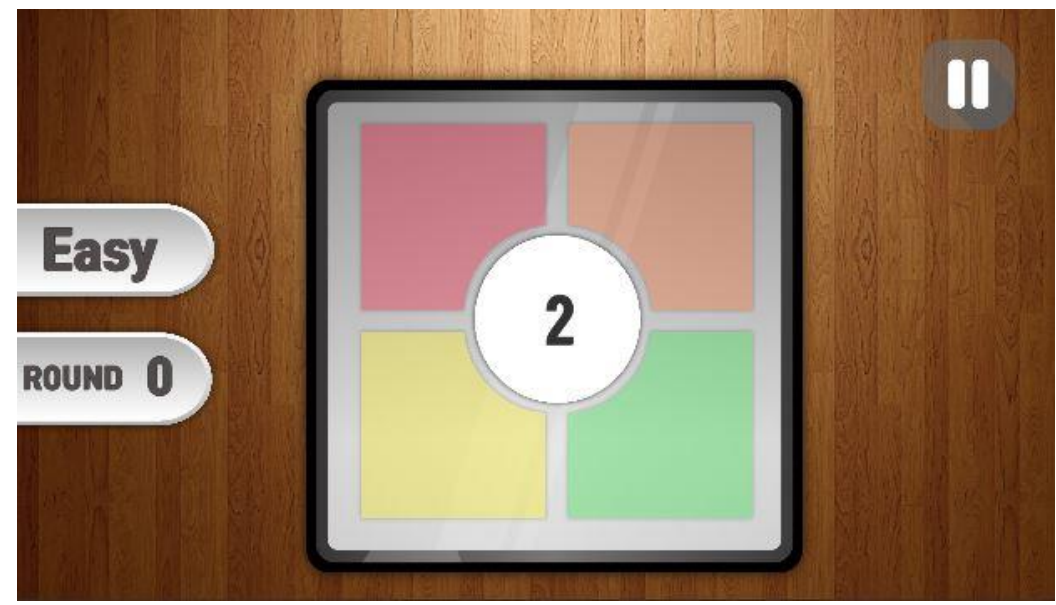

(a) "Easy Mode" Page 


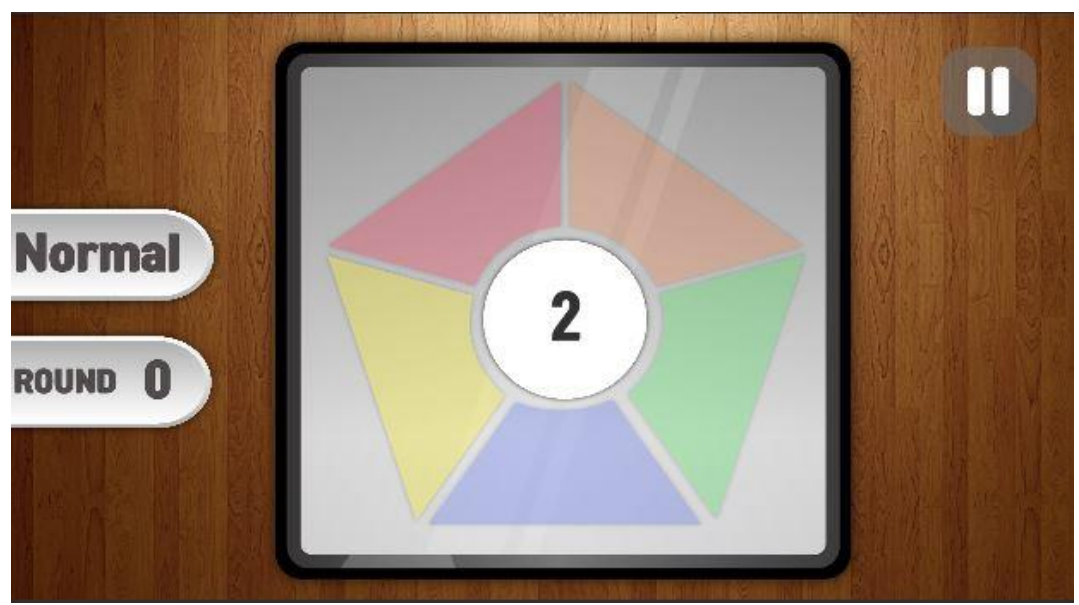

(b) "Normal Mode" Page

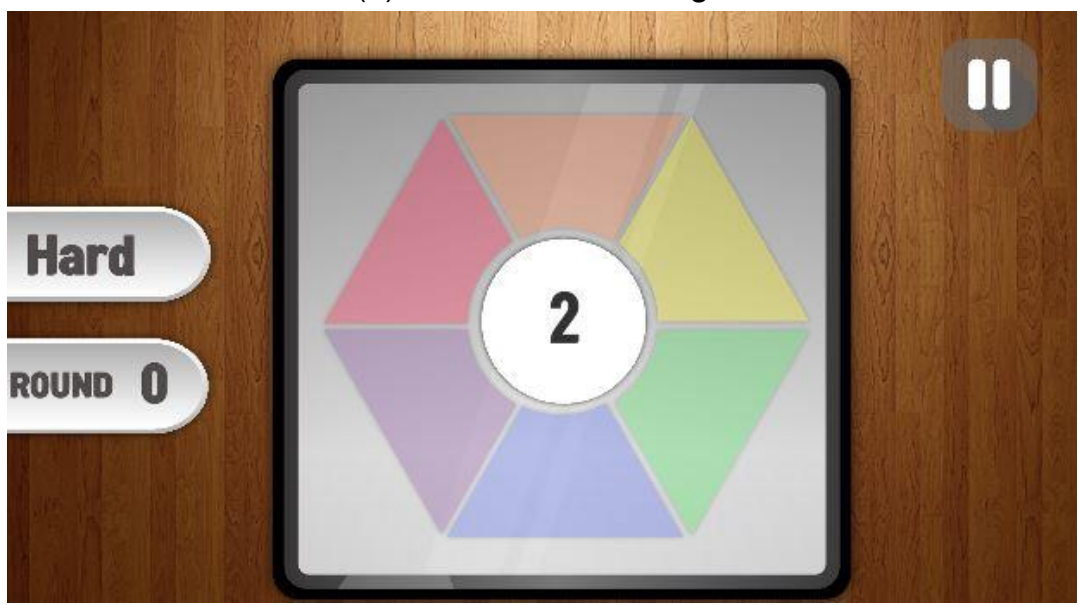

(c) "Hard Mode" Page

Figure 6. Difficulty Level-Specific Pages in the Exciting Mode

Figure 6 shows the start screens of each content difficulty level. As the level moves up, the forms change into the quadrangle, pentagon and hexagon. The number of presented space also increases along with the types of n-back in a random presentation. Such a difficulty level structure increases factors to be remembered by users and add their cognitive load.

\subsection{Content Results Page}

Figure 7 is the pages showing the results after content completion. It displays the number of successful reactions and training results according to difficulty levels. Figure 7 (a) shows the trend of changes in position-based training results among the visual stimulation-based training programs. If the Exciting tap is clicked, users can also watch the graph of changes in their corresponding training results just like in Figure 7. (a) Figure 7 (b) is the page of comparing the results of visual and auditory stimulation-based trainings. By doing so, users can compare their improvement and results in each training type to understand their areas of weakness and appropriately choose the necessary training programs for themselves. If Reply is clicked, users move to the Mode Select page in Figure 2. to re-program their training. Contents are terminated if the end button is clicked. 


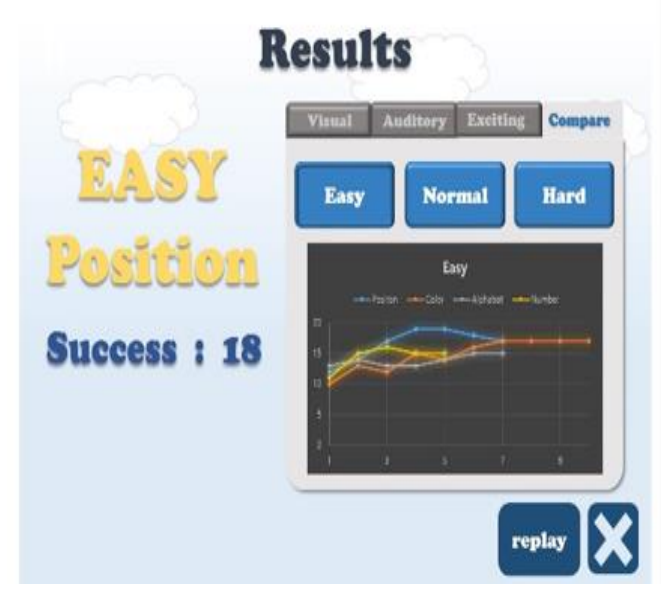

(a)

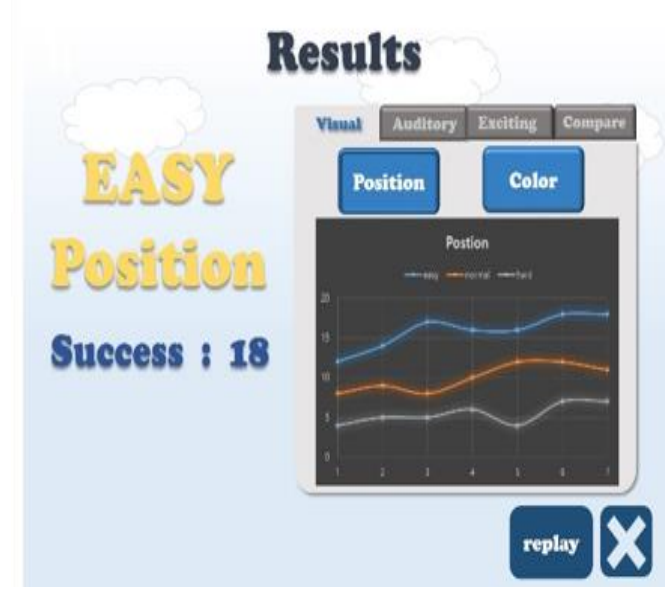

(b)

Figure 7. Content Results Pages

\section{Discussion}

This study developed a working memory training content to help improve human cognitive function based on n-back which is widely utilized as a working memory training and measurement index recently. The proposed training contents allow users to choose their desired programs between visual and auditory stimulation and provide feedback on their training results. The study program offers 4 basic n-back training contents and nback-based functional games by adding gaming elements and users can adjust their difficulty levels as they need. The developed contents and games are expected to give the following benefits to patients with cognitive disorders in training their working memory to improve their cognitive function;

First, the alphabet and number-based auditory stimulation training among the proposed 4 types of n-back contents will help activate the central executive area that controls attention. Also the visual stimulation training based on position and color will help stimulate selective attention [13]. In this manner, users can train themselves using specific stimulation to focus on the corresponding specific stimulated areas of working memory.

Second, the result page shows users their training results of each difficulty level stimulation. As users can check their training type-specific cognitive functional improvement in this page any time, they can be further motivated and feel the sense of achievement. In this manner, the program encourages their voluntary participation in training. In addition, based on their training results, users can choose an appropriate training difficulty level among the 3 different levels.

Third, users can compare the graphs of their training results of the 4 n-back contents at the same difficulty level. As they can compare results across different training types, users identify areas of insufficient training at the same difficulty level. Thus, they can understand which area of cognitive ability is poorer than others and choose to receive an intensive training for it.

Forth, since the n-back training-based functional game has more entertaining elements than other n-back training programs, it can interest users more to participate in the training. By solving randomly presented n-back tasks, users can see their utilization ability with respect to the given tasks.

To sum, the contents proposed in this paper integrates multiple existing n-back training contents for users to selectively use and informs them of the trend of their training results for users' proactive training participation as well as a higher sense of achievements and motivation. Moreover, as the program is operable via PCs and smartphones, users enjoy 
high accessibility without spatial limitation.

Future study will need to look at stroke, dementia, ADHD and other patients witha weaker cognitive function to apply the study contents and compare the correlation with existing evaluation indexes with a view to testing the effectiveness of this study training program. It will be also necessary to provide further diversified contents to broaden options for users and enable dynamic forms of training programs.

\section{Acknowledgments}

"This work was supported by the Technology Innovation Program (10062378, Development of fusion parts for 3.5 Tesla 6 channel magnetic stimulation and information feedback for a cure for intractable brain disease) funded By the Ministry of Trade, industry \& Energy (MI, Korea)".

\section{References}

[1] T. Rantanen, E. Pertti and H. Eino, "Physical activity and the changes in maximal isometric strength in men and women from the age of 75 to 80 years", Journal of the American Geriatrics Society, vol. 45, no. 12, (1997), pp. 1439-1445.

[2] A. Baddeley, "The episodic buffer: a new component of working memory", Trends in Cognitive Sciences, vol. 4, no. 11, (2000), pp. 417-423.

[3] S. Choi, "Working memory capacity, vocabulary knowledge, and reading comprehension of EFL learners", Pan-Korea English Teachers Association, vol. 25, no. 1, (2013), pp. 25-42.

[4] M. R. Dougherty and J. E. Hunter, "Hypothesis generation, probability judgment, and individual differences in working memory capacity", Acta psychologica, vol. 113, no. 3, (2003), pp. 263-282.

[5] K. A. Kiewra and S. L. Benton, "The relationship between information-processing ability and notetaking", Contemporary Educational Psychology, vol. 13, no. 1, (1988), pp. 33-44.

[6] S. Abu-Rabia, "The influence of working memory on reading and creative writing processes in a second language," Educational Psychology, vol. 23, no. 2, (2003), pp. 209-222.

[7] A. D. Baddeley and G. Hitch, "Working memory", The Psychology of Learning and Motivation, vol. 8, (1974), pp. 47-90.

[8] T. P. Alloway, S. E. Gathercole, A. M. Adams, R. Eaglen and E. Lamont, "Working memory and phonological awareness as predictors of progress towards early learning goals at school entry", British Journal of Developmental Psychology, vol. 23, no. 3, (2005), pp. 417-426.

[9] T. Klingberg, E. Fernell, P. J. Olesen, M. Johnson, P. Gustafsson, K. Dahlström, C. G. Gillberg, H. Forssberg and H. Westerberg, "Computerized training of working memory in children with ADHD-a randomized, controlled trial", Journal of the American Academy of Child \& Adolescent Psychiatry, vol. 44, no. 2, (2005), pp. 177-186.

[10] M. J. Van der Molen, J. E. H. Van Luit, M. W. van der Molen, I. Klugkist and M. J. Jongmans, "Effectiveness of a computerised working memory training in adolescents with mild to borderline intellectual disabilities", Journal of Intellectual Disability Research, vol. 54, no. 5, (2010), pp. 433-447.

[11] A. R. Conway, M. J. Kane, M. F. Bunting, D. Z. Hambrick, O. WilHelm and R. W. Engle, "Working memory span tasks: A methodological review and user's guide", Psychonomic Bulletin \& Review, vol. 12, no. 5, (2005), pp. 769-786.

[12] R. W. Engle and M. J. Kane, "Executive attention, working memory capacity, and a two-factor theory of cognitive control", Psychology of Learning and Motivation, vol. 44, (2003), pp. 145-199.

[13] R. Rodriguez-Jimenez, C. Avila, C. Garcia-Navarro, A. Bagney, A. M. de Aragon, N. Ventura-Campos, I. Martinez-Gras, C. Forn, G. Ponce, G. Rubio, M. A. Jimenez-Arriero and T. Palomo, "Differential dorsolateral prefrontal cortex activation during a verbal n-back task according to sensory modality", Behavioural Brain Research, vol. 205, no. 1, (2009), pp. 299-302.

[14] J. R. Gray, C. F. Christopher and T. S. Braver, "Neural mechanisms of general fluid intelligence", Nature Neuroscience, vol. 6, no. 3, (2003), pp. 316-322.

[15] Y. T. Lee, M. H. Kim and J. W. Son, "Effects of advancing age on drivers' cognitive workload", Journal of the Ergonomics Society of Korea, vol. 28, no. 3, (2009), pp. 73-79.

[16] S. M. Jaeggi, M. Buschkuehl, J. Jonides and W. J. Perrig, "Improving fluid intelligence with training on working memory", Proceedings of the National Academy of Sciences, vol. 105, no. 19, (2008), pp. 6829-6833.

[17] S. M. Jaeggi, M. Buschkuehl, W. J. Perrig and B. Meier, "The concurrent validity of the N-back task as a working memory measure", Memory, vol. 18, no. 4, (2010), pp. 394-412. 


\section{Authors}

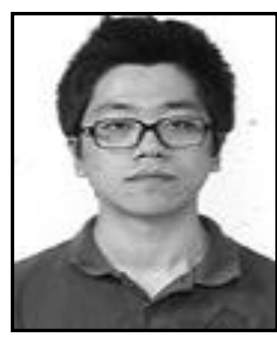

Dong-Yeon Kim, he studied Computer Engineering, and received his B.S.(2017) from Korea Polytechnic University(Korea) and his research interest is in and his research interest is in image processing, biomedical signal processing and serious game.

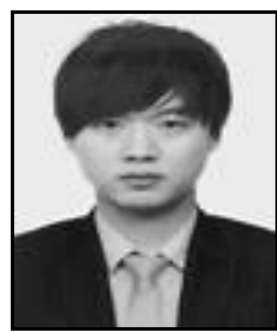

Sung-Wook Shin, he studied Computer Engineering, and received his B.S.(2011), M.S.(2013) and Ph.D. (2016) from Korea Polytechnic University(Korea). After graduation, he worked as an R\&D Engineer at Levision Co. (Korea) until 2016. In 2017, he moved to the Korea Polytechnic University (Korea) as assistant professor of Computer Engineering, and his research interest is in cognitive rehabilitation content, embedded system and serious game.

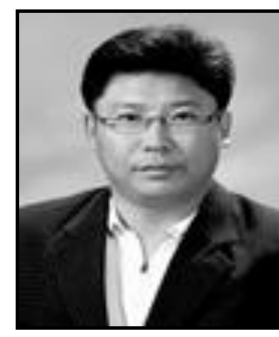

Sung-Taek Chung, he studied Electrical Engineering, and received his B.S. (1992), M.S.(1995) and Ph.D. (2000) from KAIST (Korea). After graduation, he worked as an R\&D Engineer at Medinus Co. (Korea) until 2003. In 2004, he moved to the Korea Polytechnic University (Korea) as a professor of Computer Engineering, and his research interest is in image processing, biomedical signal processing and serious game. 
International Journal of Grid and Distributed Computing Vol. 10, No. 10 (2017) 